\title{
Design and Development of Framework for Identifying Errors in Crowdsourced Map Data
}

\author{
Ramanpreet Kaur \\ PG Student \\ Guru Nanak Dev Engineering College \\ Ludhiana, Punjab, India
}

\author{
Sukhjit Singh Sehra \\ Research Scholar, IKG PTU \& Assistant Professor \\ Guru Nanak Dev Engineering College \\ Punjab, India \\ Hardeep Singh Rai \\ Department of Civil Engineering \\ Guru Nanak Dev Engineering College \\ Ludhiana, Punjab, India
}

\author{
Jaiteg Singh \\ School of Computer Sciences \\ Chitkara University \\ Punjab, India
}

\begin{abstract}
The development in the quantity of clients and the volume of data in OpenStreetMap (OSM) show the success of Volunteered geographical information(VGI) based task in attracting different types individuals from everywhere throughout the world. A huge quantity of data is produced every day by non-proficient clients and OSM faces the test of guaranteeing information quality. Since contributors have diverse perspectives about items, information integration in OSM might be considered as a type of multi-representation information combination. As due to freely availability of crowdsourced data quantity and quality assurance are two major areas to concern. This work introduces the design and development of a framework for identifying errors in crowdsourced map data, which enables volunteers to edit and tag geospatial and geographic data. Completeness, a quality parameter is used to investigate different types of errors. Initially Web-based framework is established, which includes a set of components to display the geospatial map data, indicators, markers to highlight or identify errors, as the establishment of the labeling framework. Based on this approach a prototype is developed and implemented in experiments. To actually fix the errors on OpenStreetMap, after completing the fix, it is sent back to OpenStreetMap. The result of this approach is to calculate the fixed errors by volunteers and graphically represent the stats of user contributions towards OpenStreetMap.
\end{abstract}

\section{Keywords}

OpenStreetMap, Volunteered Geographical Information, Crowdsourcing, Tags

\section{INTRODUCTION}

Crowd sourcing geographic information is an opensource geographic information which is contributed by bunches of nonexperts and gave to people in general data science in the late years. As innovation keeps on enhancing (e.g., improving the registering and geolocation abilities of hand-held gadgets) and the Internet is available by more residents, the measure of geospatial informa- tion produced by natives without formal geographic preparing is required to increase quickly.

VGI is taking after the improvement of Web 2.0 where clients are contributing in more places and more frequently. Another paramount clear expression is "crowdsourcing" which portrays VGI in business terms, connecting assets and work assignments as recommended by Howe. Harvey fought that crowdsourcing joins both data that is "contributed" and "volunteered" identifying that data that is contributed and volunteered addresses information that has been assembled without the brief learning and express decision of a man using flexible development that records territory while "volunteered" data is illustrative of information unequivocally gave. The center of this work is on crowdsourced VGI (as opposed to the AGI), and particularly on the exactness of such data. Comprehension and measuring the information nature of data gave by volunteers who may have unreported plans and/or predispositions is a critical issue in topography today. A stage towards understanding the potential data nature of volunteered data would be to evaluate key quality attributes for geospatial information that can sensibly be relied upon to be incorporated into contributed datasets, and after that be used to look at those qualities against reference wellsprings of information to measure information quality. Borderlands demonstrating and comprehension rely on upon both spatial and non-spatial information, which were hard to get before. This has constrained the advancement of borderland-related exploration. As of late, information gathering advancements have grown enormously, particularly geospatial Web 2.0 advances including online journals, distribute/subscribe, mashups, and GeoRSS, which give chances to information obtaining in borderland territories. This work introduces the design and development of a framework for identifying errors in crowdsourced map data, which enables volunteers to edit and tag geospatial and geographic data. Error data used in this work is provided by keepright [1] as primary error data. Initially web-based framework is established, which includes a set of components to display the geospatial map data, indicators, markers to highlight or identify errors, as the establishment of the labeling framework. Based on this approach a prototype is developed and implemented in experiments. To actually fix the errors on OSM, 
after completing the fix, it is sent back to OSM. The result of this approach is to calculate the fixed errors by volunteers and graphically represent the stats of user contributions towards OSM.

\subsection{Crowdsourcing}

Crowdsourcing [2] has numerous definitions in connection to the improvement of geospatial information. Brabham depicts the "methodology as utilizing online volunteers to fathom an in the past inward generation prerequisite of a business or office." Heipke recommended the term "crowdsourcing used to delineate data obtaining by vast and different gatherings of individuals utilizing web advancements.; ". Web 2.0 advances got a noteworthy change the way all sort of data is created, spread, processed, utilized and distributed. Crowdsourcing is one consequence for gathering the information which is more effectively and productively. A crowdsourcing process makes it possible to use the authority of an extensive number of clients to perform jobs at a lower expense. That is the reason these days, the term crowdsourcing more often refers to web based crowdsourcing, which compares to outsourcing employments to the Web group.

\subsection{Spatial Data}

In different fields there is a need to oversee geometric, geographic, or spatial information, which implies information identified with space. As a rule, spatial information [3, 4, 5] speaks to the area, size and state of an item on planet Earth, for example, a building, lake, mountain or township.Spatial information might likewise incorporate characteristics that give more data about the substance that is being spoken to. Spatial information are information that have a spatial part, it implies that information are associated with a spot in the Earth. A Geographic Information System coordinates equipment, programming, information, and individuals to catch, control, examine and show all types of geologically referenced data or spatial information. A GIS permits see, comprehend, counsel and translate information to uncover connections, examples and patterns. Basically spatial data includes points, polygons, LineString, CompoundCurve, CurvePolygon, CircularString, MultiPolygon, MultiLineString, MultiPoint.

\subsection{Types of Spatial Data}

In GIS, there are 2 fundamental spatial information sorts speaking to this present reality. 1) Raster information model: A representation of the world as a surface isolated into a normal matrix of cells. Raster models are valuable for putting away information that shifts consistently, as in a flying photo, a satellite picture, a surface of concoction fixations, or a height surface. 2) Vector data model : A representation of the world utilizing points, lines, and polygons. Vector models are helpful for putting away information that has discrete limits, for example, nation fringes, area bundles, and avenues.

\subsection{OpenStreetMap}

Openstreetmap (OSM) [7, 8, 9, 10] was started by Stephen Coast in July 2004 at the University College London. Since its foundation, OSM is extending scale, the quantity of enlisted clients from hundreds amidst 2004 increment to more than five hundred thousand in November 2011. As an online guide community oriented arrangement, gave intentionally by people including the catch, preparing and spread of geographic data, the undertaking points to make and appropriate vector information for the world on the grounds that most maps considered as free really have lawful or specialized limitations on their utilization, keeping down individuals from utiliz-

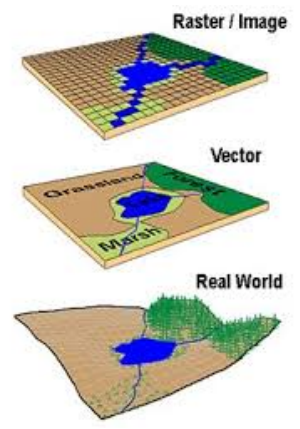

Fig. 1. Raster-Vector Information Model [6]

ing them as a part of innovative, beneficial, or sudden ways. There are three hotspots for OSM to acquire vector information, including hand-held GPS collector follow information from clients, gifts from foundation and association, vectorization of pictures, for example, Landsat, Yahoo, Imagery etc..

Spatial data is the core part of volunteered geographic information and its quality is straightforwardly identified with the exactness of spatial examination and operation. Despite the fact that OSM venture has numerous points of interest, there are worries about how the OSM quality is and what parts of utilization it can meet for the volunteers that contribute need proficient learning and adequate cartographic preparing.

\subsection{Structure of OSM data}

The OpenStreetMap information model [11] is an effective yet basic approach to speak to geographic data. Understanding the information model empowers you to associate with OpenStreetMap information in its crude frame so you can control it into arrangements that are more helpful for what you're attempting to do. You'll need to comprehend the information model in the event that you are keen on composing a guide proofreader, changing over the crude OpenStreetMap information into a configuration for use in an application, or if any of the current programming apparatuses don't give usefulness that you need.

Tag : Tags are "key-worth" sets of strings alternatively connected to each geographic component in OpenStreetMap. These labels depict the element they are joined to, and can be any pair of strings up to a greatest of 255 characters long (counting spaces), with the main limitation that keys be novel inside one component. On the off chance that there are no tags connected with a component, most renderings of the information won't show that element. In OSM Semantic information is recorded with help of tags. Tags in OSM, consist of pair of key=value; key represents features and value tells about detailed information about this given feature; e.g., highway $=$ commercial where highway stands for road or key.

Nodes : Points on Earth are called nodes and are spoken to by a scope, longitude and the same number of tags as may be proper. For instance, nodes are utilized to speak to shops, transport stops, seats, and post boxes. A hub with no labels will dependably be a subelement of another component.

Ways : A requested rundown of hubs is known as a way. A way has a most extreme of 2000 nodes to guarantee that devices and clients are not overpowered with substantial structures that are hard to control.They are utilized for speaking to direct components like trails, streets, rail lines, and electrical cables.

Relations : Relations are requested arrangements of nodes, ways or relations. Every individual from an association has an optional part 
that gives an extra bit of data about that sub element. Relations can speak to street or bike courses, turn confinements, and authoritative limits, again relying upon the arrangement of labels connected. Identifiers : Any component in an OpenStreetMap dataset, of any of the three sorts above, is recognized by a remarkable numerical id. These numbers have no other reason than to permit referencing of individual components, and have no uncommon significance. A relation or a way uses these identifiers to reference its subelements.Two ways are said to meet just on the off chance that they reference the same hub identifier, instead of two hubs with indistinguishable directions. Closed ways speaking to areas need to reference the same hub Id twice.

\subsection{Statics about OSM}

OSM statics [12] are measurements following the wonderful development of OpenStreetMap's database and group.OSM database details ordinarily naturally overhauled each day. Performs examination on the database that makes OpenStreetMap work ? demonstrates the quantity of ways, hubs, relations, and so on in the database. Tag info breaks down OSM label use.These recorded graphical diagrams beneath utilize the every day database information rundown. Stats shows in figure 2.

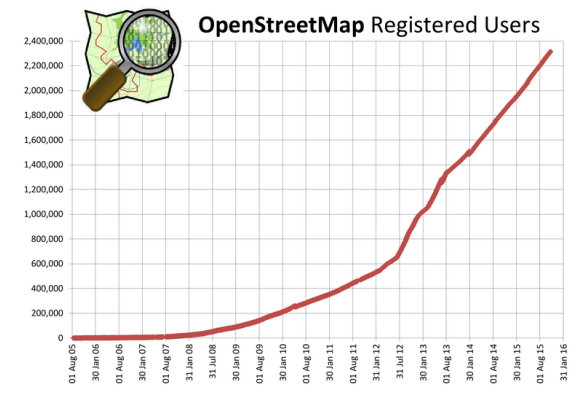

Fig. 2. Statics of Registered users [12]

\subsection{Quality Assessment of OSM}

Quality evaluation [13, 14] is a vital exploration issue in OpenStreetMap. Two major ideas, i.e. exactness and accuracy, are regularly utilized for identifying the quality of OSM data, which are semantically diverse and abused in the geospatial writing. Different quality assessment parameters that are defined in previous work are

Lineage : It records the spatial data, which incorporates the detailed information of base material from which information was extracted, the time logs of base material, and all changes involved in generating the last digital files.

Positional accuracy : It characterizes the closeness of locational data to the genuine position i.e. the outright and relative exactness of the positions of geographic components. The Absolute precision is the closeness of the direction values in a dataset to values acknowledged as or being genuine and the Relative exactness is the closeness of the relative positions of elements to their separate relative positions acknowledged as or being valid.

Completeness : It depicts the extent to which geographic components, their properties and their connections are incorporated or excluded in spatial dataset.

\section{RELATED WORK}

Xing et al. [15] has assessed that Borderlands demonstrating and comprehension rely on upon both spatial and non-spatial information, which were hard to get previously. This has constrained the advancement of borderland-related examination. As of late, information gathering innovations have grown significantly, particularly geospatial Web 2.0 advancements including websites, distribute/subscribe, mashups, and GeoRSS, which give opportunities for information procurement in borderland ranges. This paper presents the outline and improvement of a Geoweb-based labeling framework that empowers clients to label and alter land data.We first set up the GeoBlog model, which comprises of an arrangement of geospatial segments, posts, pointers, and remarks, as the establishment of the labeling framework. GeoBlog is executed such that online journals are squashed up with OpenStreetMap.In addition, author show a change to existing distribute/subscribe frameworks with backing for spatio-worldly occasions and memberships, called Spatial Publish/Subscribe, and additionally the occasion office system for steering messages from the distributers to the endorsers. A model framework taking into account this methodology is actualized in investigations.

Liu et al. [16] has assessed that Web 2.0 empowers a two route collaboration in the middle of servers and customers. GPS recipients get to be accessible to more nationals and are usually found in vehicles and advanced cells, empowering people to record and share their direction information on the Internet and alter them on the web. OpenStreetMap (OSM) makes it feasible for residents to add to the procurement of geographic data. He thought about the utilization of OSM information to discover recently mapped or fabricated streets that don't exist in a reference guide and make its redesigned rendition. For this reason, a dynamic buffering system is proposed for deciding an ideal cushion range to distinguish the new boulevards in the OSM information. In the accompanying step, the identified new streets are converged into the reference guides geometrically, topologically, and semantically. Tries different things with OSM information and reference guides over a region of $8494 \mathrm{~km} 2$ in the city of Wuhan, China and five of its $5 \mathrm{~km} 5 \mathrm{~km}$ regions are led to exhibit the achievability what's more, feasibility of the system. It is exhibited that the OSM information can include $11.96 \%$ or a sum of $2008.6 \mathrm{~km}$ of new streets to the reference guides with a normal accuracy of $96.49 \%$ and a normal review of $97.63 \%$.

Camboim et al. [17] has assessed that the joining of customer produced substance made in a community oriented environment is in effect progressively considered an important data to reference maps, even from authority map organizations, for example, USGS and Ordnance Survey.In Brazil, many years of absence of venture has brought about a topographic guide scope that is both obsolete and unequally circulated all through the domain. In this paper author planed to break down the spatial dissemination of overhauls of OpenStreetMap in provincial and urban regions in the nation to comprehend the examples of client redesigns and its relationship with other monetary and formative variables.

Zielstra et al. [18] has assessed that The kind of information an individual benefactor adds to OpenStreetMap (OSM) changes by locale. The nearby learning of an information giver takes into consideration the gathering and altering of nitty gritty components, for example, little trails, park seats or fire hydrants, and also including trait data that must be gotten to locally. Rather than this, satellite symbolism that is given as foundation pictures in OSM information editors, for example, ID, Potlatch or JOSM, encourages the commitment of less nitty gritty information through on screen digitizing, in many cases for zones the patron is less acquainted with. 
Knowing whether a region is a piece of a patron's home locale or not can consequently be a helpful indicator of OSM information quality for a geographic district. Author investigates the altering history of hubs and routes for 13 exceptionally dynamic OSM individuals inside of a two-layered bunching procedure to depict an individual mapper's home locale from remotely mapped ranges. The discoveries are assessed against those found with a formerly presented technique which decides a supporter's home locale singularly taking into account spatial grouping of made hubs. Hecht et al. [19] has assessed that Because of money related or regulatory requirements, access to official spatial base information is at present constrained to a little subset of every single potential client in the field of spatial arranging and research. This builds the helpfulness of Volunteered Geographic Information (VGI), specifically OpenStreetMap (OSM), as supplementary datasets or, at times, option wellsprings of essential information.As opposed to the OSM road system, which has as of now been altogether examined and observed to be for all intents and purposes complete in numerous regions, the level of fulfillment of OSM information on structures is still hazy. In this paper author portray strategies to examine building culmination and apply these to different test regions in Germany. The outcomes demonstrate that unit-based culmination measurements(e.g. total number or zone of structures) are very touchy to incongruities in displaying between authority information and VGI. In this manner, author prescribe article based routines to ponder the fulfillment of OSM building foot shaped impression information. An examination from November 2011 in Germany showed a fulfillment of $25 \%$ in the government conditions of North RhineWestphalia and $15 \%$ in Saxony. Albeit further examinations from 2012 affirm that information culmination in Saxony has ascended to $23 \%$, the rate of new information data was moderating in the year 2012 .

\section{METHODOLOGY}

\subsection{Design and Implementation}

An idea of framework is created that gets the client's present area with the help of geolocation API and get longitude and latitude of user's current location and recovered the information from OSM server with the assistance of leaflet (a javascript library) and draw a bounded box to display the map in this bounded box, utilizes diverse markers to appear and highlight mistakes to clients with various styles. Different parts and steps used to outline this structure are said beneath.

\subsection{Authorisation}

Initial step is to verify the client by login or signup to Openstreetmap server.OAuth [20] on OpenStreetMap is an instrument is utilized which permits clients to approve outsider applications to do things with their OSM client account without that application taking care of the client's secret key.Rest API v0.6 is used as third party to login with Openstreetmap server. Distinctive conventions used to enrolling application as OAuth consumers are :

Prior to an application can utilize the OAuth convention to increase approved access to the secured assets on the OSM server it must be enlisted as OAuth consumer.

Name : This is the presentation name of the application. Which will be introduced to the client.

Main Application URL : This is a data URL with data about the application. It isn't identified with the OAuth convention by any means. At the point when a client approves access from your application on the OSM site, the OSM site will show a link.
Callback URL : Subsequent to enrolling a web application we can enter a callback URL the OSM site will summon divert the client toward the end of the OAuth handshake. The URL is discretionary. In this structure, for callback URL, url is defined, which recover the map information from postgresql database.

Support URL : Final step is support URL, which is optional.

Handle Errors : The errors callback function, if gave while calling getCurrentPosition() or watchPosition(), expects a PositionError object as its first parameter.

\subsection{Basic protocols authorization with openstreetmap API}

Step 1 : After registering application supply CONSUMER-KEY + CUSTOMER-SECRET into it.

Step 2 : When user runs application request token url will called and oauth_token $+\mathrm{s}$ oauth_token_secret will receive.

Step 3: After this point it diverts the client to Authorize URL + '?oauth_token=' + authorizetoken

Step 4 : client sign in on OpenStreetMap.org, the site requests that they concede consents

Step 5 : After this it redirects to callback URL which is provided while registering.

Step 6 : Access token and its mystery are utilized for further correspondence

Algorithm used to get login token from OSM server to perform various functionalities on OSM server is explained below :

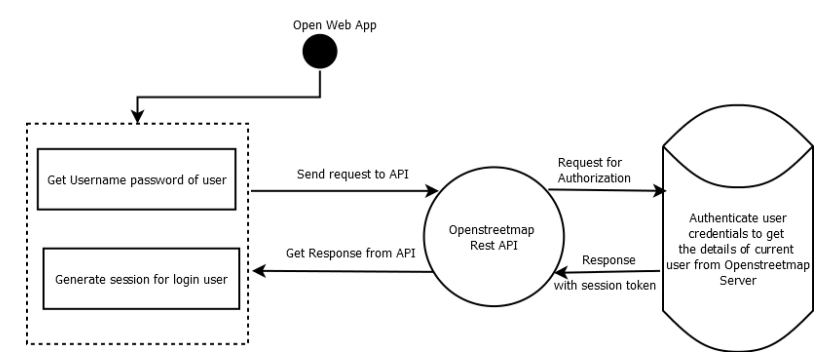

Fig. 3. Authorize User

subsectionPresentic map data to users Share Geo Location [21] : The geolocation API permits the client to give their area to web applications on the off chance that they so seek. For security reasons, the client is requested authorization to report area data. The geolocation API is distributed through the guide, geolocation object. To get the client's present area, getCurrentPosition() system is utilized.This starts a non concurrent solicitation to recognize the customer's position, and request the arranging equipment to get avant-garde data. At the point when the position is resolved, the characterized callback function is executed. To detect user's current location following steps are used.

Check for Compatibility : Compatibility is checked by testing for the vicinity of the geolocation object.

Decide the User's Current Location : getCurrentPosition() will asynchronously cover the client's present area. Call it when the page loads, so it will effectively populate and put something aside for some other time - the beginning position :

Leaflet is used to get data from openstreetmap server and build the map in given bounded box.Leaflet, a broadly utilized open source 


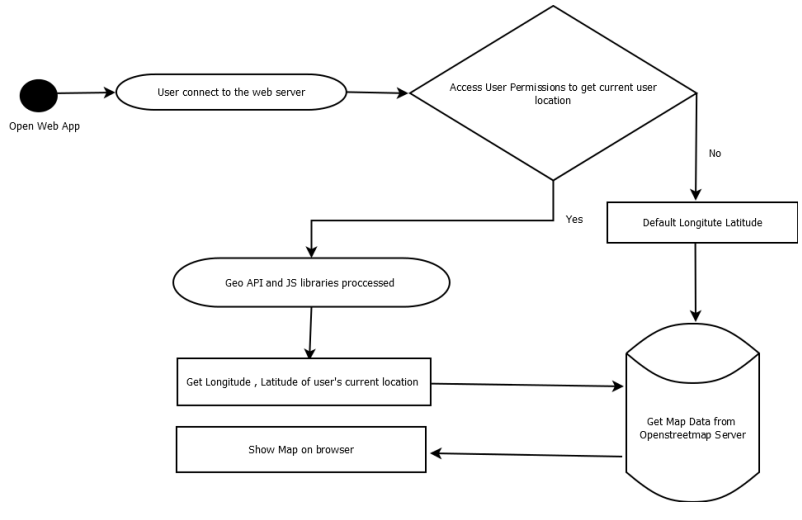

Fig. 4. Access Current Geo Location

JavaScript library is utilized to assemble this web mapping applications. It ties a Leaflet "map" component to a HTML component, for example, a div.Different elements like raster type, vector, grouped, controls perform their different roles. When Openstreet map data is loaded in browser leaflet, a js library is loaded to manage data. Data coming from server got filtered with an algorithm to extract those tag and nodes which have errors.

Different Layers are used to build map data. Two sorts of layers while building map data : Base Layer and Overlay Layer.This distinction controls a few parts of how client connect with an OpenLayers Map. Base Layer : Base Layers are fundamentally unrelated layers, which means one and only can be empowered at any given time. The as of now dynamic base layer decides the accessible projection (coordinate framework) and zoom levels accessible on the map. In this system base layer is proposed with two different styles which can change on run time by user by selecting particular one. In this project to styles : Grey style, Streets are used for base layer. Gray style is used to simple shows the cities names and point tags on the other hand streets styles provide the detailed ways with street style.

Non Base/Overlay Layer : Non base layers some of the time called overlays are the different option for Base Layer. Numerous non-base layer can be empowered at once. These layers don't control the zoom levels of the guide, yet can be empowered or incapacitated at specific scales by $\mathrm{min} / \mathrm{max}$ scale/determination parameters with the goal that they are just empowered at a specific level. Layers used to build map are : pack of layers you need to join into a gathering to handle them as one.Following code has used to create base layer.

Filter Error : The main challenge to identify the errors in map data retrieved from OSM server. Map data having JSON format is filtered to get the tags having errors and are displayed with different styles and markers. Bootstrap style sheet is used to fill the styles in markers. Algorithm used to filter errors is :

\subsection{Edit Map}

After generating map or geoJSON data in given bounded box editing functionality is provided to fix the errors by volunteers and after fixing these errors changes are sent back to Openstreetmap to actually fix the Openstrretrmap data. In this project errors defined by keepright are used as base errors. Major errors considered in project are : (1) Missing Tags (2) Ways without node (3) Worship Places without religion (4) Multiple nodes on same point (5) Rail-

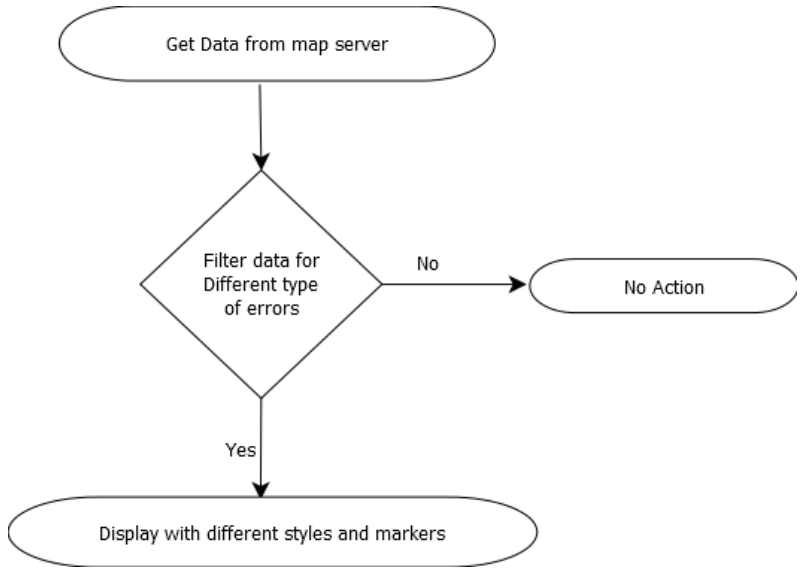

Fig. 5. Filter Errors

way crossing without tag (6) Intersection without junction (7) Point of intersect without name

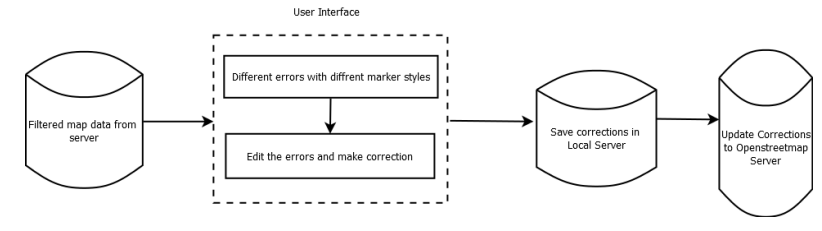

Fig. 6. Interface to edit errorss

Algorithm is used to filter above errors and these are highlighted with different markers having different styles defined with bootstrap. Leaflet provide layer group and layer controls to perform different functionality.

Layers Control : Leaflet has a decent little control that permits your clients control what layers they need to see on your map. Click events on markers provide the edit option and detailed information about that tag or point. After clicking on edit option user can edit that error and information entered by user is updated in openstreetmap server.

Upload OSM File : Uploading OSM is another feature added to this work, shows the map data of users's own choice. OSM file can be uploaded, algorithm is used to read the content of uploaded file and display the map of given data. Next step to filter data for errors and highlight errors with markers and different styles. Pointers are used to points the errors and are highlighted with markers. Click events are used to show details of given node or tag. Following is the pseudo code of the algorithm used to upload the file to OSM data.

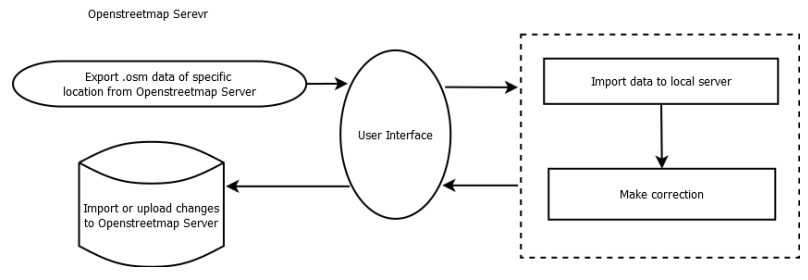

Fig. 7. Upload specified error file 


\section{RESULTS}

After the long implementation, the got application can be acknowledged through its settled interface and its completed functionalities. Major concern of this work is that crowdsourced map data, due to its openly access functionality has concern about its accuracy and quality and quantity assurance. The first objective of this work is to investigate the major errors in OSM data. In this work major 10 type of errors are investigated and explained. In this work errors in the selected region of Ludhiana city is considered. Errors investigated in this works are : Missing tags; Ways without node; Worship Places without religion; Multiple nodes on same point; Railway crossing without tag; Intersection without junction; Point of intersect without name;. From different type of quality access parameters completeness parameter is the basic concern of this project. In this investigation aprox. 1000 nodes were found having completeness errors in the given area.

Second map objective to design the framework layout to displayed these investigated errors to user. In this work bootstrap and different libraries are used to figure out these errors. Different errors are displayed with different markers and points to distinguish between these errors. User can update or edit the errors of their concern. Multiple users at a time can contribute towards fixation of these errors. A layout is provided which gives the functionality to the user to edit and update the errors. With this work multiple users at the same time can give their contribution towards the completeness of OSM data. After fixing the errors these are sent back to OSM server for modification in actual data. Initially this algorithm found approximate 1000 no errors and after contribution of volunteers this figure from all sources and all existing frameworks it has been reduced to $50 \%$. In this way this work puts some efforts towards the quality assurance of crowdsourced volunteered data.

With the help of user contribution graph given in this work provides the stats of daily users contributing data towards the OSM. It gives the graphs on user interest and contribution towards crowdsourced data. It provides the graph of current user and most active users, which motivate and encourage the users to contribute theirs efforts towards the completeness of crowdsourced data.

\section{Experimental Results}

As in the given fig. 8 it provides the user to identify their self to get the track of contribution towards the OSM data. The given figure provides the layout to access user's identity to track the user's contribution.

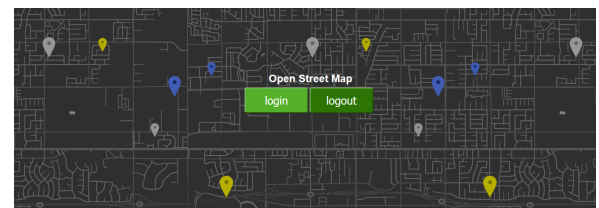

Fig. 8. Authorization of user from Server

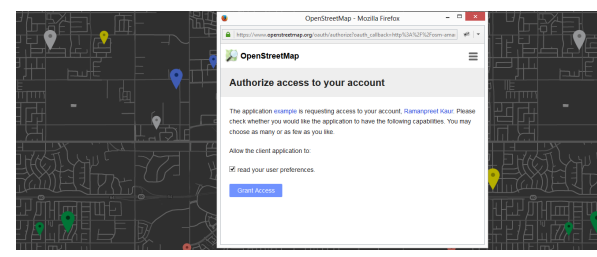

Fig. 9. Take grant from user to share information
As in fig. 9 the errors investigated during this work. Different types of errors are represented with markers and different style and formats. Markers with red labels are show in this figure points to missing values.

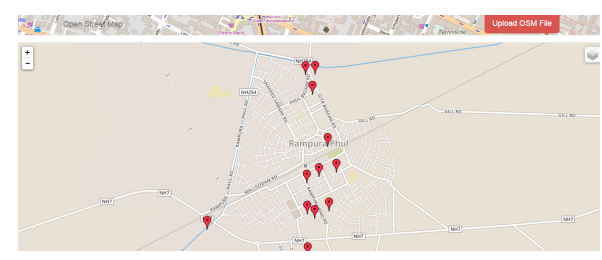

Fig. 10. Investigate errors in crowdsourced map data

Fixation of errors is major aspect of this work. In this work edit option is provided to user to fix this error. User is provided with the click events to alter these given error tags. As provided in the figure 10 edit link give the pointer to fix this tag. On the other hand figure 11 provides the layout to edit the tag. It takes the tag info from user and to fix it in actual database OSM, sent back to the OSM server.

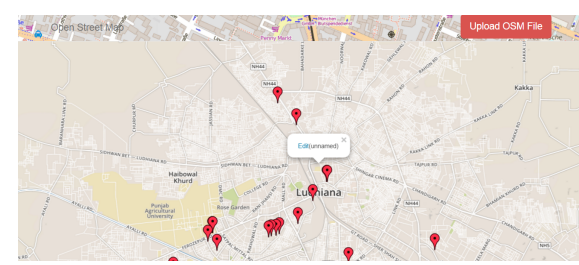

Fig. 11. Pointer to edit the error

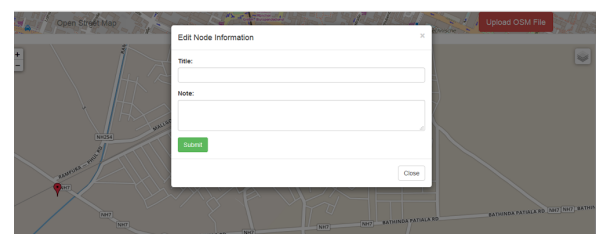

Fig. 12. Edit Tag or Node

\section{DISCUSSION}

In this work errors in crowdsourced map data are investigated and represented with different styles and designs and fixed back to actual data of OSM server. As Pascal Neis et al. in 2012 analyze the user's contribution of VGI project. As in that work author accessed the contribution of all the users according to time and location towards volunteered geographical map data. In that work author has compared the user's interest and contribution between registered used carried only one activity and the active users in contrast in this proposed work contribution of given user in different time interval is graphically represented. With OSM data many games have been implemented to focus on quality concern of crowdsourced data e. kort game was designed to increase quality assurance. In this work framework is provided to edit the tags and points but is does not give any stats of user contribution towards VGI. In the proposed 
system user can view or edit the map data of his own wish whereas in previous work user was not provided with that option.

\section{CONCLUSION}

This work introduces a framework to identifying the errors in crowdsourced map data. In this work the main objective is to investigate the different errors and warnings in Volunteered geographical data. In this work users can contribute towards map data freely. In this project it is concluded that data contributed by different types of volunteers, having different passions, skills and knowledge. So the data quality and quantity assurance remains the main concern of crowdsourced data. OpenStreetMap is the biggest example of crowdsourced data. So quality assurance is the basic concern about OpenStreetMap data and in this project completeness is considered as a basic parameter of quality assurance to investigate the errors. Next step in this work is to represent these errors with with styles and formats and users can edit or modify these tags to fix these errors i.e this framework provide an approach to individuals to contribute towards OSM data by editing and modifying the tags. After fixing these errors these changes are sent back to OSM server to actually alter the OSM data. After fixing the errors to OSM server results are validated and analyzed to check the completeness. This frameworks also provide the options to see the errors of their own choice by uploading the .osm file exported from OSM server so that they can edit and view the errors of the area of their own choice. In this approach user's contribution and stats towards the crowdsourced map data or OSM data is represented graphically.

\section{FUTURE SCOPE}

Completeness and accuracy are the major aspects in VGI. So in future, there are the different aspects to enhance the tagging framework to improve quality and quantity on crowdsourced data ara: 1) Ontology can be extending to make it more adaptable and reasonable. 2) by enhancing the interface/client configuration to enhance client cognizance; 3 ) by performing huge examinations to assess framework ease of use and the nature of the information it gives.

\section{REFERENCES}

[1] Keep Right - OpenStreetMap Wiki. URL http://wiki. openstreetmap.org/wiki/Keep_Right [Accessed Mar. 02, 2016].

[2] Thierry Buecheler, Jan Henrik Sieg, Rudolf M. Fchslin, and Rolf Pfeifer. Crowdsourcing, Open Innovation and Collective Intelligence in the Scientific Method-A Research Agenda and Operational Framework. In ALIFE, pages 679-686, 2010.

[3] Daniel Cintra Cugler, Dev Oliver, Michael R. Evans, Shashi Shekhar, and Claudia Bauzer Medeiros. Spatial Big Data: Platforms, Analytics, and Science. GeoJournal, 2013.

[4] Jae-Gil Lee and Minseo Kang. Geospatial big data: challenges and opportunities. Big Data Research, 2(2):74-81, 2015.

[5] Mustafa Neamah Jebur, Zahra Ziaei, Mahyat Shafapour Tehrany, and Abdul Rashid Mohamed Shariff. A review of recent developments in national spatial data infrastructures (NSDI).

[6] GIS Spatial Data Types. URL http://gis .washington. edu/phurvitz/professional/SSI/datatype.html [Accessed Feb. 11, 2016].
[7] OpenStreetMap - Wikipedia, the free encyclopedia. URL https://en.wikipedia.org/wiki/OpenStreetMap [Accessed Feb. 16, 2016].

[8] Jamal Jokar Arsanjani, Alexander Zipf, Peter Mooney, and Marco Helbich. An Introduction to OpenStreetMap in Geographic Information Science: Experiences, Research, and Applications. In OpenStreetMap in GIScience, pages 1-15. Springer, 2015.

[9] Peter Smith and Peter Mooney. OSM-CAT: A Java tool for OpenStreetMap Contributor Analysis.

[10] Louai Alarabi, Ahmed Eldawy, Rami Alghamdi, and Mohamed F. Mokbel. TAREEG: a MapReduce-based system for extracting spatial data from OpenStreetMap. In Proceedings of the 22nd ACM SIGSPATIAL International Conference on Advances in Geographic Information Systems, pages 83-92. ACM, 2014.

[11] Mihai Codescu, Daniel Couto Vale, Oliver Kutz, and Till Mossakowski. Ontology-based Route Planning for OpenStreetMap. In Terra Cognita 2012 Workshop, page 62, 2012.

[12] Stats - OpenStreetMap Wiki. URL http://wiki. openstreetmap.org/wiki/Stats. [Accessed Mar. 15, 2016].

[13] Sukhjit Singh Sehra, Jaiteg Singh, and Hardeep Singh Rai. Assessment of OpenStreetMap data-A review. arXiv preprint arXiv:1309.6608, 2013.

[14] Mohammad Forghani and Mahmoud Delavar. A Quality Study of the OpenStreetMap Dataset for Tehran. ISPRS International Journal of Geo-Information, 3(2):750-763, May 2014.

[15] Hanfa Xing, Jun Chen, and Xiaoguang Zhou. A GeowebBased Tagging System for Borderlands Data Acquisition. ISPRS International Journal of Geo-Information, 4(3):15301548, August 2015.

[16] Changyong Liu, Lian Xiong, Xiangyun Hu, and Jie Shan. A Progressive Buffering Method for Road Map Update Using OpenStreetMap Data. ISPRS International Journal of GeoInformation, 4(3):1246-1264, July 2015.

[17] Silvana Camboim, Joo Bravo, and Claudia Sluter. An Investigation into the Completeness of, and the Updates to, OpenStreetMap Data in a Heterogeneous Area in Brazil. ISPRS International Journal of Geo-Information, 4(3):1366-1388, August 2015.

[18] Dennis Zielstra, Hartwig Hochmair, Pascal Neis, and Francesco Tonini. Areal Delineation of Home Regions from Contribution and Editing Patterns in OpenStreetMap. ISPRS International Journal of Geo-Information, 3(4):1211-1233, November 2014.

[19] Robert Hecht, Carola Kunze, and Stefan Hahmann. Measuring Completeness of Building Footprints in OpenStreetMap over Space and Time. ISPRS International Journal of GeoInformation, 2(4):1066-1091, November 2013.

[20] OAuth - OpenStreetMap Wiki. URL http://wiki. openstreetmap.org/wiki/OAuth. [Accessed Feb. 17, 2016].

[21] Using geolocation - Web APIs | MDN. URL https://developer.mozilla.org/en-US/docs/ Web/API/Geolocation/Using_geolocation] [Accessed Feb. 15, 2016]. 